\title{
Secular and cycle variations of polar activity of the Sun
}

\author{
V.I. Makarov ${ }^{1}$, A.G. Tlatov ${ }^{2}$ and D.K. Callebaut ${ }^{3}$ \\ ${ }^{1}$ Pulkovo Astronomical Observatory, St.Petersburg, Russia, email: makarov@gao.spb.ru \\ ${ }^{2}$ Kislovodsk Solar Station of the Pulkovo Observatory, Russia, email: solar@narzan.ru \\ ${ }^{3}$ Physics Dept., UA, University of Antwerp. B-2610, Antwerp. Belgium \\ email: dirk.callebaut@ua.ac.be
}

\begin{abstract}
An analysis of polar activity of the Sun was carried out using the observations in the optical range. The secular and cycle peculiarities of the magnetic activity on the high latitudes were found:

1. an increase of the area of polar caps of the Sun, occupied by unipolar magnetic field at the minimum activity during 1878-2000;

2. a decrease of coronal temperature at the high-latitude zones during the last 50 years;

3. the connection between the polar and sunspot activities;

4. outset and duration of the polar activity cycle;

5. drift of the activity to the poles and from the poles after the magnetic field reversal.
\end{abstract}

In the absence of a long-term record of magnetograph measurements of the solar magnetic field the investigation of polar activity of the Sun in the last 120 years has been based on the latitude-time distribution of unipolar areas of the large-scale magnetic field during 1878-2000. The unipolar regions are evident on magnetograms. At the boundaries of unipolar regions where the radial component of the magnetic field is zero, $H_{\alpha}$ prominences and dark filaments are observed. They form the pattern of the so-called magnetic neutral lines, Fig. 1.

I. We calculated the area of polar zones of the Sun, $A_{p z}$, occupied by unipolar magnetic field on $H_{\alpha}$ synoptic magnetic charts from 1878 to 1996 . We found a gradual decrease of the annual minimum latitude of the high-latitude zone boundaries, $\Theta_{2 m}$, of the global magnetic field of the Sun at the minimum of activity from $53^{\circ}$ in 1878 down to $38^{\circ}$ in 1996, Fig. 2., yielding an average decrease of $1.2^{\circ}$ per cycle, (Makarov et al. (2002)).

We compared $<A_{p z}>_{11}$ with the $<a a>_{11}$ and the Wolf number $<W>_{11}$, Fig. 3.: $<A_{p z}>_{11} \approx 0.16<W>_{11}+9.3 ; \quad<A_{p z}>_{11} \approx 0.84<a a>_{11}+22.5$; $<A_{p z}>_{11} \approx 60.8 \cdot\left(1-\sin \Theta_{2 m}\right) ; \quad<a a>_{11} \approx 0.19<W>_{11}+8.1$;

Here we use the correlation between the geomagnetic $<a a>_{11}$ index and polar cap areas $<A_{p z}>_{11}$ to obtain an estimate of the latitude of the high-latitude zone boundary $\Theta_{2 m}$ in the Maunder Minimum. Using "11-year" average of index we obtained:

$<a a>_{11} \approx 1.2<A_{p z}>_{11}-3.0 ; \quad \sin \Theta_{2 m} \approx-0.014<a a>_{11}+0.96$.

Using these estimates one can find that the polar cap areas of the Sun $\left\langle A_{p z}\right\rangle_{11}$ correspond to the value of $\Theta_{2 m} \approx 60^{\circ}$ in Maunder Minimum, Fig. 4.

It is known that Earth's surface temperature is correlated with the geomagnetic $<a a\rangle_{11}$ index. For the period 1880-2000 we found:

$<T>_{11} \approx 0.039<a a>_{11}-0.88 ; \quad<T>_{11} \approx 1.8^{\circ}-2.9^{\circ} \sin \Theta_{2 m}$

We can extrapolate to the Maunder Minimum to infer the solar induced temperature 


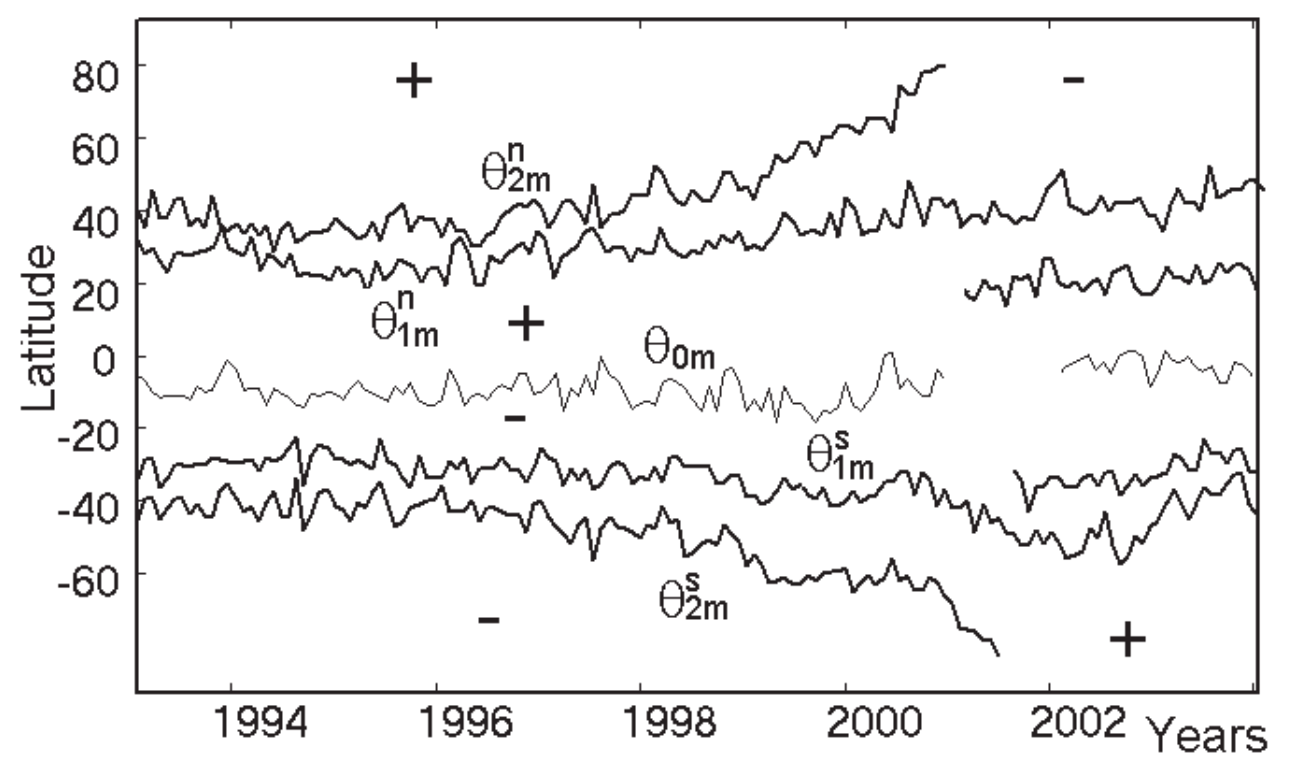

Figure 1. Solid lines represent migration trajectories of magnetic neutral lines (or the zone boundaries with the opposite polarities on either of its sides in the order $+/-$ or $-/+$ ) of the large-scale magnetic field derived from $H_{\alpha}$ synoptic charts in the Northern $\left(\Theta_{1 m}^{n}, \Theta_{2 m}^{n}\right)$ and Southern $\left(\Theta_{1 m}^{s}, \Theta_{2 m}^{s}\right)$ hemispheres for the period 1993-2003. The latitude $\Theta_{0 m}$ is the equator boundary.

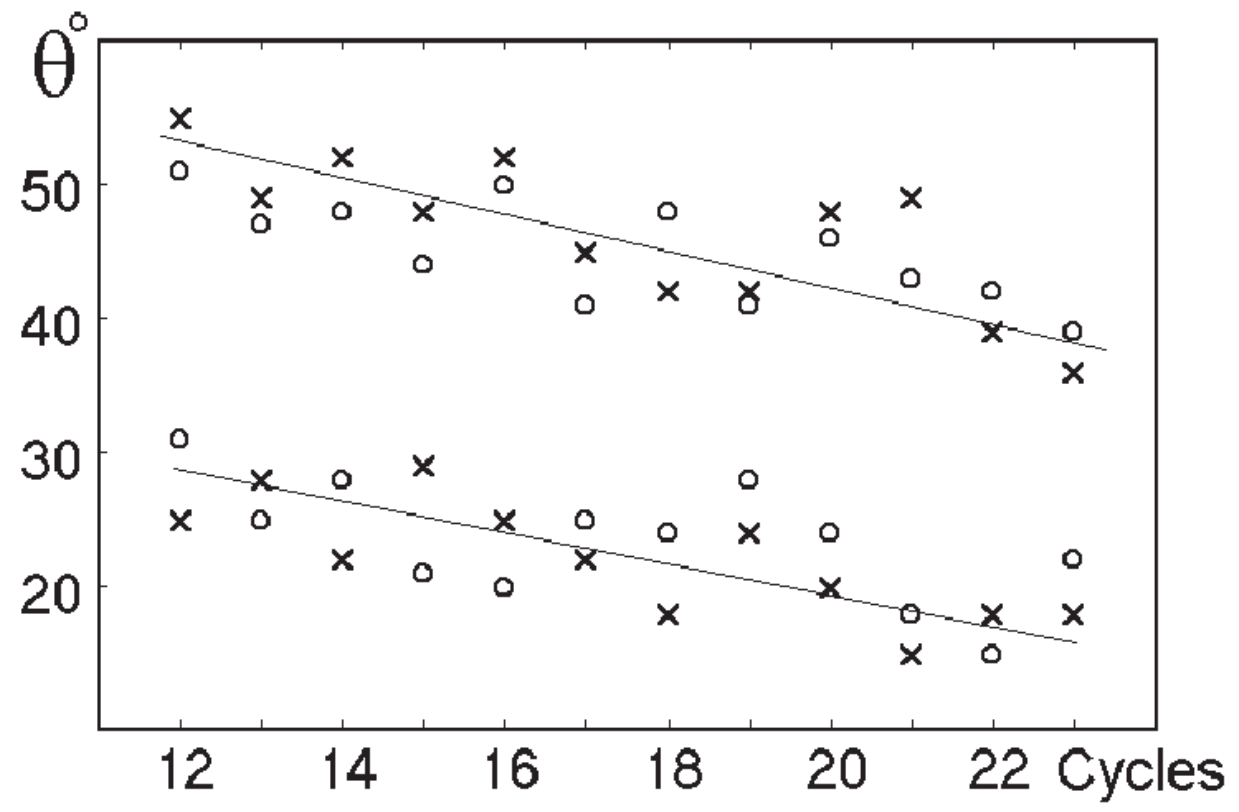

Figure 2. Annual mean latitude of the high latitude $\Theta_{2 m}$ (upper part) and low latitude $\Theta_{1 m}$ (lower part) zone boundaries of the large-scale magnetic field of the Sun ( $\mathrm{x}-\mathrm{N}$ and o-S hemishere) during the minimum activity of 11-year cycles $12-23$ according to $H_{\alpha}$ magnetic synoptic charts for $1878-1996$. 


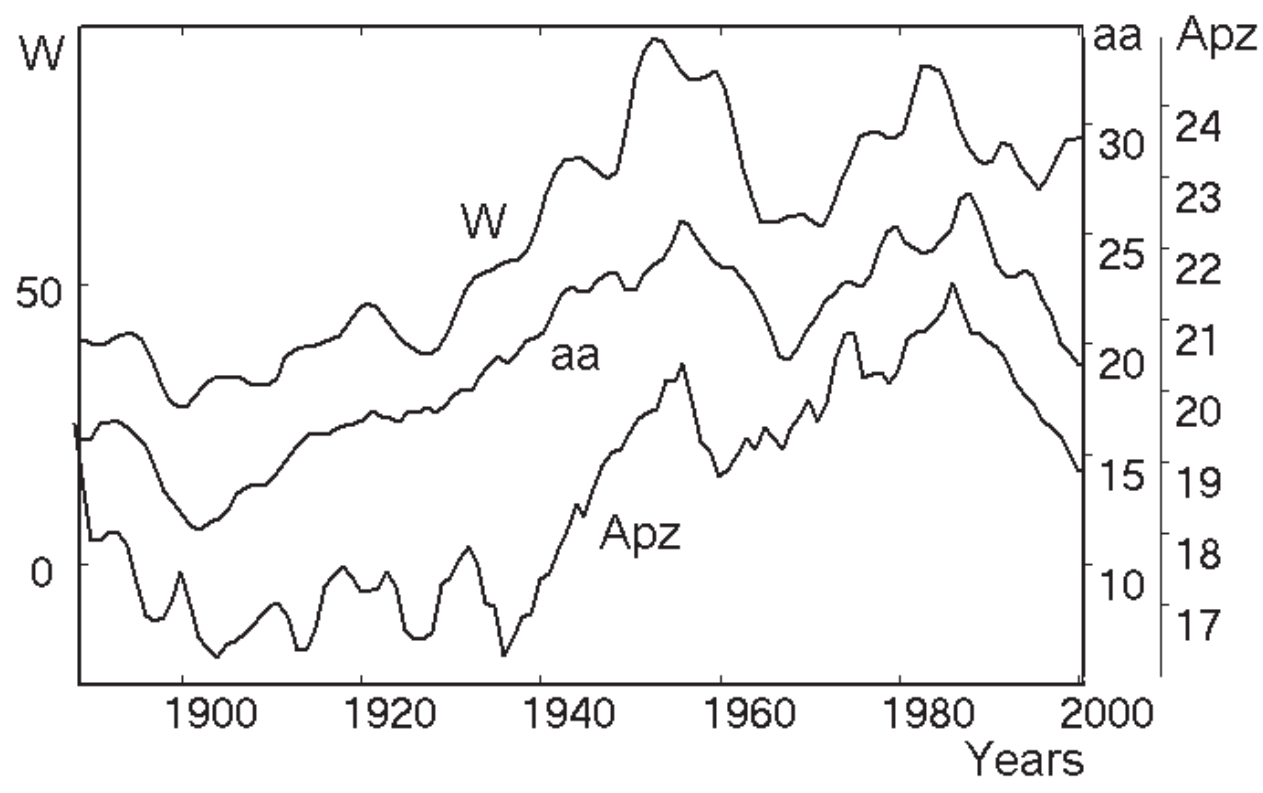

Figure 3. The continuous curve $\left\langle A_{p z}\right\rangle_{11}$ represents the run of mean annual area of unipolar magnetic regions of polar caps of the Sun, averaged with 11-year smoothing, during 1880-2001. The upper and middle curves represents of the Wolf numbers $\langle W\rangle_{11}$ and geomagnetic index $<a a>_{11}$ averaged with 11-year smoothing.

change. We obtained estimates for the temperature deficit during the Maunder Minimum $\left(-1.0^{\circ}\right)$ relative to the present $\left(\approx 0^{\circ}\right)$, yielding an increase of $1.0^{\circ}$.

II. It is possible to assume, that an increase of the polar cap area $\left\langle A_{p z}>_{11}\right.$, occupied by unipolar magnetic field of the Sun, led to an increase of the area of polar coronal holes. We assume that an increase of polar magnetic flux from the Sun was connected with an increase of the areas of polar corona holes. This phenomenon may explain an increase of the high-latitude coronal ratio $K I_{6374} / K I_{5303}$ and hence, a decrease of coronal temperature by $0.1 \cdot 10^{6} \mathrm{~K}$ at the high-latitude zones in the minimum sunspot activity during 1957 - 2002, Fig. 5. These global effects are connected with the long-term changes of the inner structure of the Sun, to the waxing and waning of the Sun's activity in conditions like Maunder Minimum and, probably, to the global warming of the Earth besides greenhouse warming, ocean-atmosphere coupling, etc.(Makarov et al. (2004))

III. The secular decrease of the latitude of a zone boundary between the polar and sunspot activities was found. It was shown that the annual mean latitude in the minimum activity decreases from $53^{\circ}$ in 1878 down to $38^{\circ}$ in 1996 . Here we draw attention on the consequences of a doubling of the area of the Sun's polar caps. It is well known that the Sun's polar magnetic field is a manifestation of the poloidal field of the solar dynamo, where as the sunspot areas are a toroidal field. It was found the correlation between the polar cap area $\left\langle A_{p z}\right\rangle_{11}$ (or polar magnetic flux) and the sunspot areas $\left\langle S_{s p}\right\rangle_{11}$ (or toroidal magnetic flux) during the 120 years from 1880 up to 2000 (Makarov et al. (2003)). This correlation is: $\left\langle A_{p z}\right\rangle_{11} \approx 4 \cdot 10^{-3} \cdot S_{s p}+15$, here $\left\langle A_{p z}>_{11}\right.$ in $10^{21} \mathrm{~cm}^{2}$, $<S_{s p}>_{11}$ in $10^{-6}$ of the area of hemisphere. 


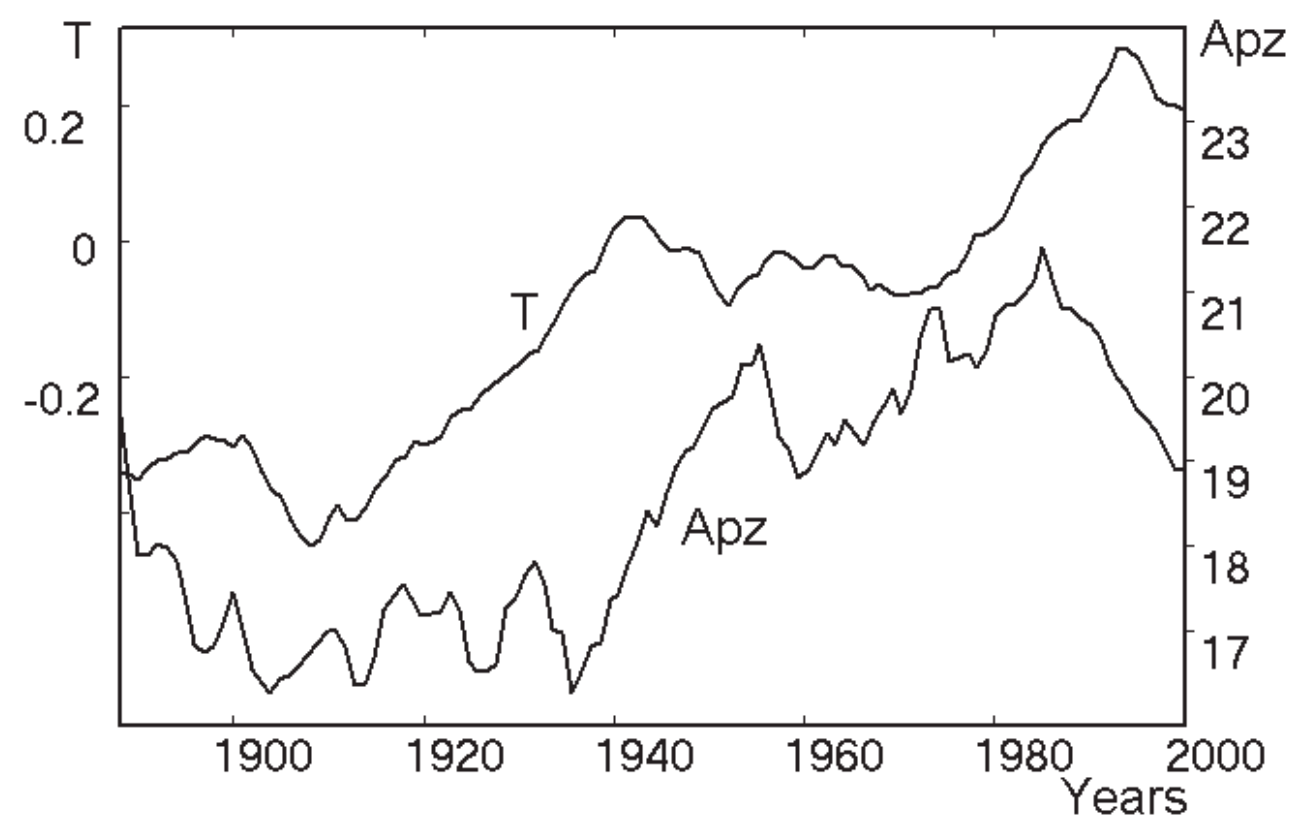

Figure 4. Comparison of the Sun's polar cap area occupied by unipolar magnetic field $\left\langle A_{p z}>_{11}\right.$ and the variation of the global surface temperature of the Earth $\langle T\rangle_{11}$ over the last 120 years for $1880-2000$.

IV. We report evidence to show that the magnetic flux elements of the size and of the polarity sign as the polar faculae makes their appearance at the latitudes around $40^{\circ}$ $45^{\circ}$ as early as $4-5$ years before the epoch of the polar reversal. The pattern that provides this evidence is derived from the Kitt Peak magnetic observations for the period 19752000. In Fig. 6 we have plotted the latitude - time distribution of $B^{2}$ contours, where $B_{i}$ the magnetic flux in the sunspots. The number of "+" polarity pf flux elements $\left(n^{+}\right)$as a fraction of the total number of such elements of either polarity, i.e. $n^{+} / n^{+}+n^{-}$) shown by the contours in the gray shaded areas. The contour levels are $0.55,0.60,0.65$ and so on. This plot shows that the polar faculae cycle commences almost simultaneously with the commencement of the sunspot cycle (Makarov et al. (2003a)).

$\mathrm{V}$. We found the two latitude drifts of the K-CaII activity during the polar cycle. The first type is related to the pole ward migration of the K-CaII activity in the beginning of polar cycle, and the second one connected with the equator ward migration from poles in the direction to the low latitudes after the polar magnetic field reversal, Fig. 7.

We have digitized and processed the daily K-CaII-line full disk spectroheliograms from the archive of the Kodaikanal Observatory during 1907-1995. At the high latitudes the K-CaII bright points form a polar branch of solar activity at the period between the polar magnetic field reversals. This polar activity shows both 11-year's and 22-year's cycles. We found that the polar K-CaII bright point cycles proceed on average 5.5 years the sunspot cycles, Fig. 8. Contrary to the sunspot activity, the intensity of the even cycles of polar activity exceeds the intensity of the even sunspot cycles. The polar magnetic field reversals have occurred in the $5.8 \pm 0.6$ years after the minimum the K-CaII activity. The correlation coefficients were calculated by shifting the polar faculae cycle relative to the sunspot cycle by \pm 10 years. The maximum correlation coefficients were obtained 


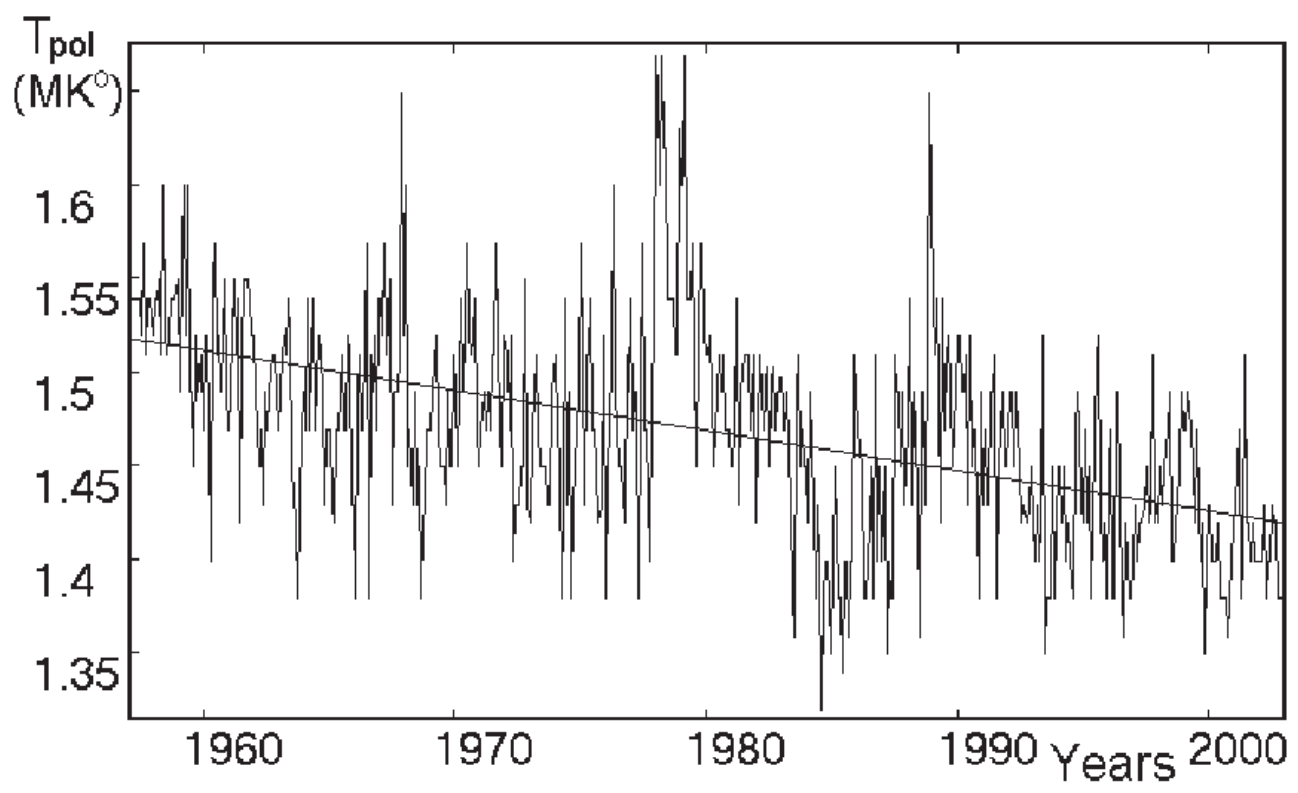

Figure 5. The change of the high-latitude coronal temperature Tpol (in $10^{6} K^{\circ}$ ) during 1957-2002 according to the ratio $K I_{6374} / K I_{5303}$. It is seen a decrease $\left\langle T>_{\text {pol }}\right.$ by $0.1 \cdot 10^{6}$ $\mathrm{K}$, although real temperature of the corona at the high-latitude zones changes from $1.6 \cdot 10^{6} \mathrm{~K}$ to $1.3 \cdot 10^{6} \mathrm{~K}$.

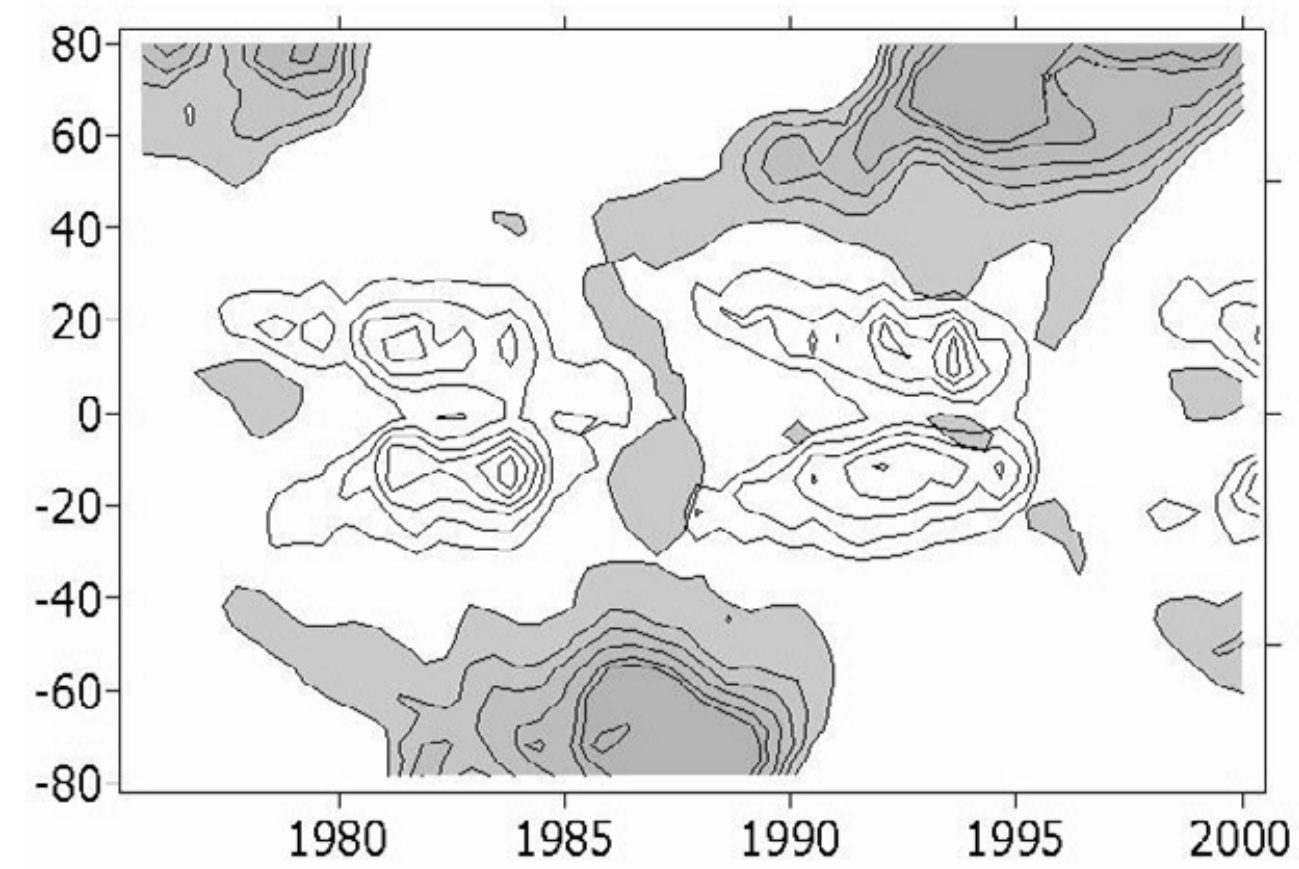

Figure 6. The sunspot diagrams are the latitude-time distribution of the value $B^{2}$. The polar faculae diagrams (pf) are the latitude-time distribution of the bright points of "+" polarity of the magnetic field relatively to all bright points, or the value $n^{+} /\left(n^{+}+n^{-}\right)$at the latitudes more that $40^{\circ}$. One can see that the polar faculae and sunspot cycles begin at the same time. 


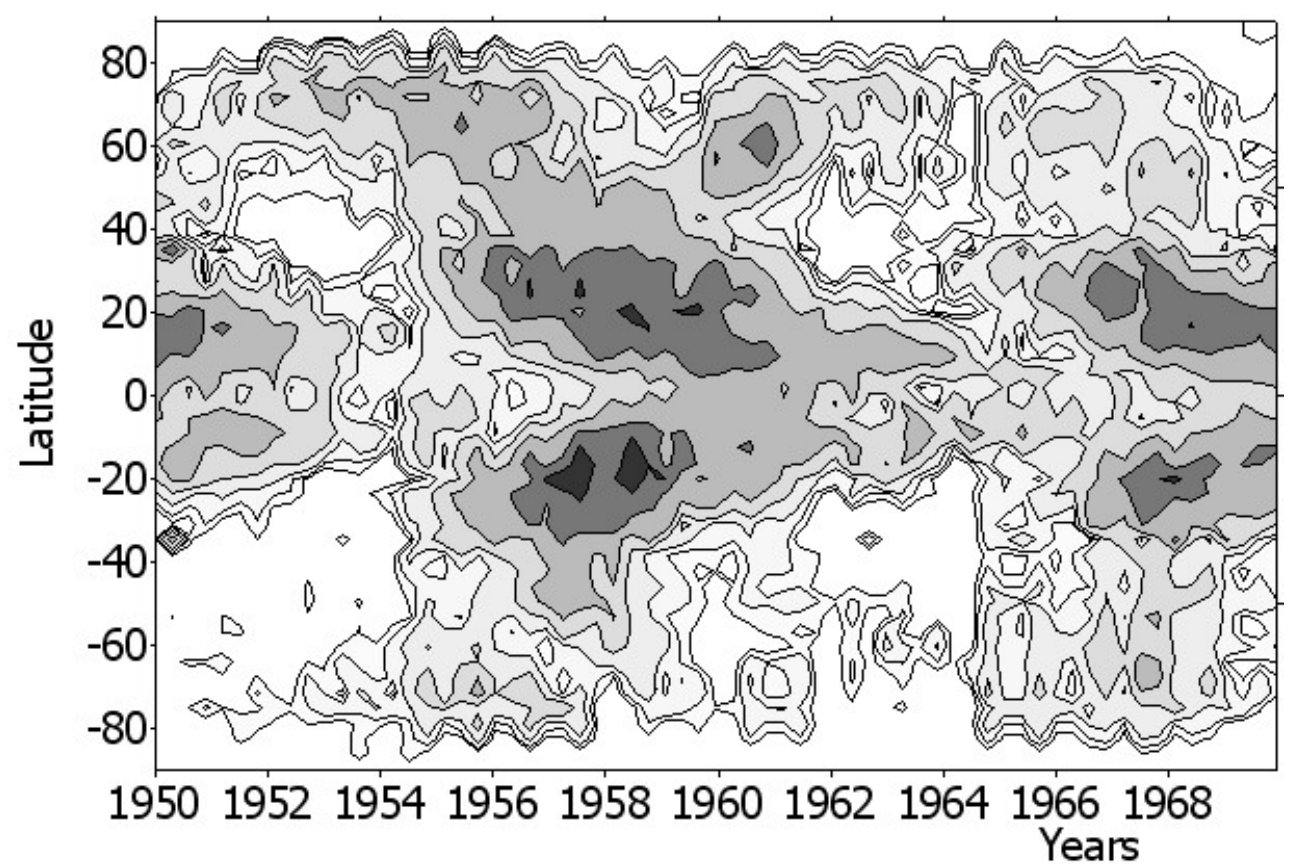

Figure 7. The latitude-time distribution of the bright K-CaII points with the area S:(100-500) $\cdot 10^{-6}$ of the hemisphere. Two latitude drifts of the K-CaII activity during the polar cycle are present.

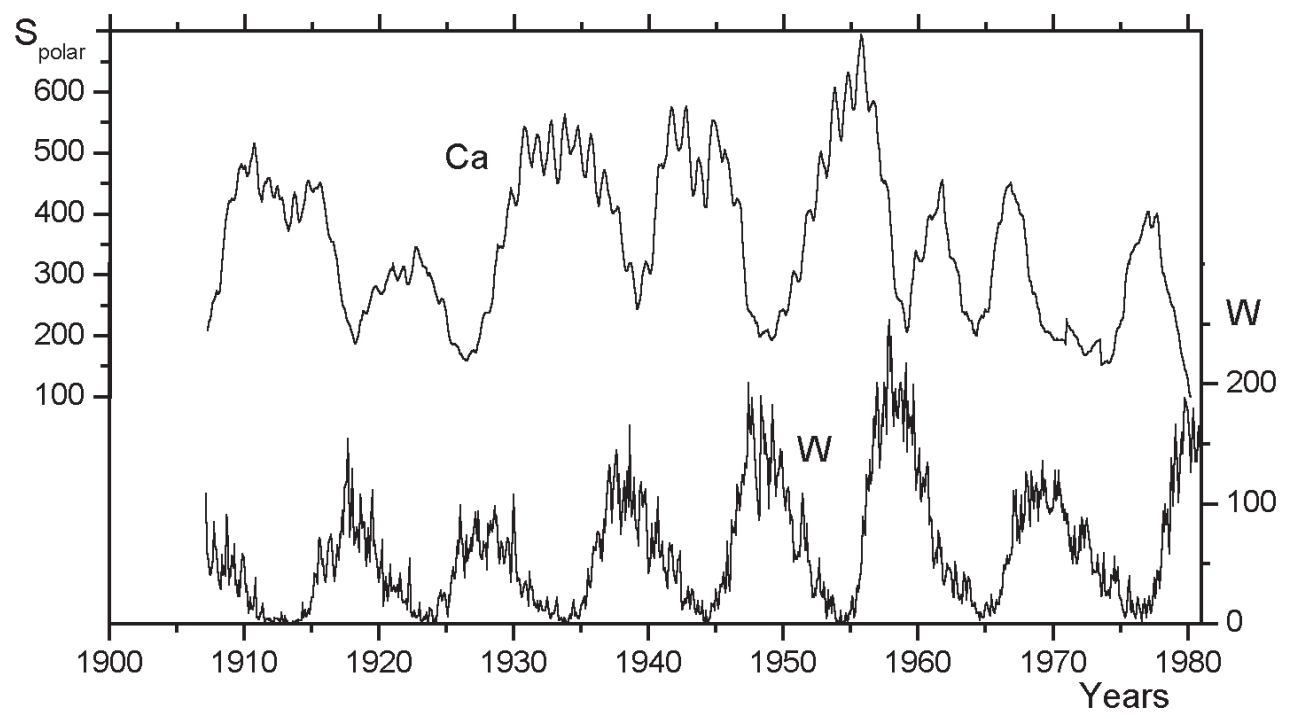

Figure 8. The mean monthly area of the K-CaII bright points at the latitudes above $70^{\circ}$ of the northern hemisphere and Wolf number, $\mathrm{W}(\mathrm{t})$.

with a shift of $5.7 \pm 0.3$ year for the cycles $20-22$, although for the cycle 23 it equals 7.6 years (Makarov \& Makarova (1996)).

Before we have used $H_{\alpha}$ synoptic charts for the last 9 cycles of the solar activity from 1915 to 1999. It was shown that the cycles of the large-scale magnetic field of the Sun 
precede on the average by 5.5 years the sunspot activity cycles, W(t), (Makarov et al. (2001), see Figure 1). This means that the weak large-scale magnetic fields of the Sun do not result from decay and diffusion of strong fields from active regions as it is assumed in all modern theories of the solar cycle.

VI. One of the puzzling features of solar activity is the way the polar magnetic fields cancel and reversals take place. It is commonly believed that the magnetic activity of the Sun is generated by the dynamo operating in a thin layer at the base of the convection zone. The toroidal field is believed to be generated by the action of differential rotation on poloidal field. Dynamic processes responsible for the cycle reversal of the poloidal and toroidal fields are not fully understood.

An interesting feature soon after the polar field reversal is the high-latitude solar activity that manifests in the form of polar faculae. The recognition that the polar faculae are an important component of the global cycle is gaining strength as more observations on these structures accrue (Makarov \& Sivaraman K.R.(1990); Makarov \& Makarova (1996)). They possess kilogauss magnetic fields (Homann et al. (1998)). In the highresolution K-CaII line scans, the polar faculae are seen as small emission features at the boundaries of the network cells. It means that the source flux for the polar faculae is the flux brought out by the ephemeral active regions.

Another aspect pertains to the filament bands and their poleward migration. The filaments represent the neutral lines that divide the unipolar magnetic regions of opposite polarities. They demonstrated the excellent agreement between the polar reversals obtained from the filament bands and those from the magnetograms. Following this, they worked out the epochs of polar field reversals over a period of 12 solar cycles (1872-2001) (Figure 1 of Makarov \& Sivaraman (1989)) and (Figure 1 of Makarov et al. (2001a)).

Magnetic field reversal at the north and south poles of the Sun is the phenomenon that imparts the cyclic nature to the solar cycle phenomenon on the Sun. The epoch of the polar field reversal marks the culmination of one solar cycle and the beginning of the next one and is an important parameter that defines the duration of the polar activity, needed for the understanding of the mechanism of the solar cycle. It was shown that the epoch of the last disappearance of the polar crown filament band alone represents the true epoch of the polarity reversal of the polar fields and this heralds the beginning of the new solar cycle. On the $H_{\alpha}$ images of the Sun the filaments represent the neutral lines that divide uni-polar regions of opposite polarities. The filament bands act as good tracers to chronicle the temporal evolution of the unipolar magnetic regions at the high latitudes. As soon as the filament bands have reached the respective poles, the polar activity of the new cycle begins to to appear in the form of polar faculae at latitudes more than $40^{\circ}$.

$H_{\alpha}$ synoptic charts have been constructed for the period 1880 - 2003 from various sources, (Makarov et al. (2003b)). We have determined the epochs of the polar field reversals in the low degree l-modes from two independent data series - one, using the $H_{\alpha}$ synoptic charts and the second, using Stanford synoptic magnetic field data, (Makarov et al. (2003b)). We constructed the latitude-time diagram that shows the epochs when the polemost filament bands (or the crown filaments) reach the respective poles and cause the polar field reversals. A 3-fold reversal of the polar magnetic field was discovered. It was observed in one of the hemispheres (Makarov \& Sivaraman (1989)).

It is known that the solar activity at latitudes greater $40^{\circ}$ manifests as polar faculae and ephemeral active regions at the photosphere, as bright points in the CaII K-line, as dark points in the HeI-line $10830 \mathrm{~A}^{\circ}$ in the chromosphere, and as XPB's and coronal holes in the corona and so on. After every polar reversal the regions above $40^{\circ}$ latitudes show polar faculae. The number of polar faculae reaches a maximum during the years 
of minimum sunspot activity. The global solar activity in any cycle has two components in each hemisphere: the high latitude component consisting of the polar faculae and the second component is the sunspot phenomenon. The new cycle shows up first as faculae at high latitudes soon after the polar reversal and leads the sunspot phenomenon by 5-6 years. Each of these components has a 11-year duration. Thus the two components belonging to the same cycle occur in the two latitude zones on each hemisphere, the polar faculae cycle, leading the sunspot cycle by 5-6 years (see Figure 4 of (Makarov \& Sivaraman K.R.(1990)).

It is the practice to use the length of the sunspot cycle for the estimation of many parameters of the solar-terrestrial relations. It is obvious the polar activity of the Sun has a dominant role in deciding the structure and behavior of the interplanetary magnetic field. It is clear from the present study that might be appropriate to use the length of the polar cycle in preference to the length of the sunspot cycle in such studies.

\section{Acknowledgements}

This research was supported by the Russian Fund for Basic Research, grants 02-0216035 and 03-02-16091 and the National Program "Astronomy".

\section{References}

Makarov V.I. \& Sivaraman K.R. 1989 Solar Phys. 119, 35.

Makarov V.I. \& Sivaraman K.R. 1990 in J.O.Stenflo (ed.), Solar Photosphere: Structure, Convection and Magnetic fields, IAU Symp. 138, 281.

Makarov V.I. \& Makarova V.V. 1996 Solar Phys. 163, 267.

Homann, T., Kneer, F., \& Makarov, V.I. 1998 Solar Phys. 175, 81.

Makarov V.I., Tlatov A.G., Callebaut D.K., Obridko V.N. \& Shelting, B.D. 2001 Solar Phys. 198, 409.

Makarov V.I., Tlatov A.G. \& Sivaraman K.R. 2001a Solar Phys. 202, 11.

Makarov V.I., Tlatov A.G., Callebaut D.K. \& Obridko V.N. 2002 Solar Phys. 206, 383.

Makarov V.I., Tlatov A.G. \& Callebaut D.K. 2003 Astron. Nachr. 324, 381.

Makarov V.I., Tlatov A.G. \& Sivaraman K.R. 2003a Astron. Nachr. 324, 382.

Makarov V.I., Tlatov A.G. \& Sivaraman K.R. 2003b Solar. Phys. 214, 41.

Makarov V.I., Tlatov A.G. \& Callebaut D.K. 2004 Solar Phys. in press. 